'Servicio de Hematología. Hospital General de México "Dr. Eduardo Liceaga" Ciudad de México, México.

2Laboratorio de Biología Molecular, Servicio de Hematología. Hospital General de México. "Dr. Eduardo Liceaga" Ciudad de México, México. ${ }^{3}$ Laboratorio de Inmunogenómica y Enfermedades Metabólicas, Instituto Nacional de Medicina Genómica, Secretaria de Salud, Ciudad de México, México.

Fuente de Apoyo: Proyecto apoyado por la Dirección de Investigación Hospital General México HGM/DI/08/204/04/17, D1/15/103/03/57.

Recibido el 29 de agosto de 2017, aceptado el 5 de julio de 2018.

Correspondencia a: Adolfo Martínez Tovar Laboratorio de Biología Molecular del Servicio de Hematología. Hospital General de México "Dr. Eduardo Liceaga" Ciudad de

México. 06726, México. mtadolfo73@hotmail.com

\section{Efecto de la metformina en la etapa de inducción en pacientes con leucemia aguda linfoblástica y su impacto clínico en la supervivencia}

\author{
CHRISTIAN RAMOS PEÑAFIEL ${ }^{1}$, IRMA OLARTE CARRILLO ${ }^{2}$, \\ RAFAEL CERON MALDONADO ${ }^{2}$, ENRIQUE MIRANDA PERALTA ${ }^{2}$, \\ ETTA ROZEN FULLER ${ }^{1}$, JUAN JULIO KASSACK IPIÑA ${ }^{1}$, \\ FEDERICO CENTENO CRUZ ${ }^{3}$, JUAN COLLAZO JALOMA ${ }^{1}$, \\ ADOLFO MARTÍNEZ TOVAR ${ }^{2}$
}

\section{Effect of metformin added to chemotherapy on the survival of patients with acute lymphoblastic leukemia}

Background: Metformin has antineoplastic and cancer protective effects in vitro, sensitizing leukemia cells to chemotherapeutic agents, inducing apoptosis and cell cycle arrest. Aim: To assess the effect of metformin on the induction stage in patients with ALL and its impact on overall survival and relapse. Material and Methods. We included 123 patients treated with metformin and without metformin. The dose used was $850 \mathrm{mg} P O$ at $8 \mathrm{~h}$ intervals. The survival analysis was used by Kaplan-Meier method, the difference between the distinct groups was performed using the log Rank test. Results. The overall survival at a median follow up of 700 days of follow-up was $43 \%$, with a disease-free survival of $47 \%$. Regarding the treatment groups, patients with metformin had a lower rate of relapse compared to the group receiving only chemotherapy (6.5\% vs $17.1 \%$, $p=0.006)$. Conclusions. The addition of metformin to the conventional treatment of ALL was associated with an improvement in survival, this association being independent of the type of biological risk at diagnosis.

(Rev Med Chile 2018; 146: 846-853)

Key words: Metformin; Precursor Cell Lymphoblastic Leukemia-Lymphoma; Therapeutics.
E 1 tratamiento moderno de la leucemia linfoblástica aguda (LLA) incluye la combinación de diversas drogas (esteroides, antraciclinas, alcaloides de la vinca, asparaginasa), y más recientemente el uso de diferentes estrategias de inmunoterapia como lo son los fármacos anti-CD20 (rituximab), anticuerpos bi-específicos (ByTE), o mediante las estrategia de anticuerpos quiméricos (CART) ${ }^{1-2}$. A pesar de que la mayor experiencia del tratamiento de la LLA fue obtenido de los protocolos pediátricos, el pronóstico en pacientes adultos continúa siendo adverso (30-40\% de supervivencia a largo plazo), e inclusive peor en aquellos casos que hayan presentado una recaída principalmente al año del diagnóstico (10\% de supervivencia a largo plazo $)^{3-5}$. Dentro de estos fármacos, solamente blinatumomab es el que ha mostrado mejores resultados en comparación con la quimioterapia (RC, $34 \%$ vs $16 \% \mathrm{p}<0,001)^{6-7}$. Desafortunadamente, en países en vías de desarrollo el limitado acceso de este fármaco al igual que su costo eleva- 
do (213,600 USD por dos ciclos de inducción), lo vuelven aún una estrategia poco accesible, tanto para aquellos que no cuentan con un donador HLA-compatible o aquellos que no puedan ser enrolados en algún ensayo clínico ${ }^{8,9}$. Paralelo a esta investigación, diversos grupos han evaluado la eficacia anti neoplásica de diversos fármacos de uso cotidiano y cuya indicación original no era el tratamiento del cáncer (claritromicina, valproato de magnesio, ácido acetil salicílico, estatinas). Dentro de estos, tal vez el más estudiado hasta el momento es el clorhidrato de metformina (1,1-dimetilbiguanida clorhidrato $)^{10-15}$. Posterior a las primeras observaciones en pacientes diabéticos en Tayside, Escocia (7,3\% de cáncer en los pacientes que utilizaron metformina en comparación con $11,6 \%$ con otro tipo de terapia, OR: $0,63<0,53-0,75)$, y seguida de otros estudios observacionales en pacientes diabéticos ${ }^{16,17}$, son diversos los mecanismos antineoplásicos o protectores que se le han adjudicado a metformina, siendo los principales la activación de AMPK (proteincinasa activada por AMP), el bloqueo de la vía de proliferación P13K-AKT-mTOR, y más recientemente la supresión de HIF-1, glicoproteína-P y $\mathrm{MRP}^{18-20}$. Estos últimos mecanismos han sido descritos en líneas celulares de hepatocarcinoma en el bloqueo de la expresión de los genes de resistencia a drogas (glicoproteína-P), mediante el bloqueo de la expresión del factor nuclear kappa-B (NFk-B $)^{21-22}$. En leucemias agudas, Pan y colaboradores identificaron en la línea celular de leucemia (REH) que la adición de metformina $(500 \mu \mathrm{M})$, mejora la sensibilidad de diferentes fármacos utilizados durante la mayoría de los esquemas terapéuticos (daunorrubicina, etopósido, L-asparaginasa, vincristina), mientras que otros fármacos como la insulina (glargina o aspartato), incrementan la quimioresistencia ${ }^{23}$. Debido a esto, en nuestro centro se evaluó la adición de metformina al esquema de quimioterapia convencional para el tratamiento de la LLA del adulto, logrando un incremento en el porcentaje de remisiones completas (RC), pero de manera más importante, en la reducción en el porcentaje de recaídas tempranas ( $25 \%$ vs $47,9 \%)^{24}$. En este estudio se presentan los resultados del seguimiento a largo plazo de los pacientes portadores de LLA de precursores B (cromosoma Ph negativo) a los que se les adicionó metformina al tratamiento convencional de LLA.

\section{Material y Métodos}

\section{Pacientes}

Se estudiaron pacientes con diagnóstico de leucemia linfoblástica aguda de estirpe B corroborada mediante citometría de flujo, considerándose los marcadores de superficie (CD19, CD22, CD10, CD20, IgMc, IgMs), excluyendo aquellos casos con leucemia de linaje T o leucemias linfoides con algún tipo de marcador mieloide. Se excluyeron aquellos casos con alteraciones moleculares como BCR-ABL1 o cariotipos de mal pronóstico, al igual que aquellos pacientes con infiltración al sistema nervioso central al diagnóstico.

\section{Esquema de tratamiento}

El esquema utilizado para el tratamiento fue el HGMLAL07, el cual se basa en un pretratamiento con esteroides semejante al protocolo GIMEMA ALL0288, una etapa de inducción la remisión mediante tres fármacos (antraciclinas, alcaloides de la vinca y esteroides), una etapa de consolidación que incluye la protección a sistema nervioso central mediante metotrexato y una etapa de mantenimiento con duración de 2 años (Tabla 1). Todos los pacientes que se encontraba en tratamiento con metformina, la mantuvieron durante la etapa de inducción a la remisión, solo en caso de un evento adverso se redujo la dosis a $850 \mathrm{mg}$ PO una vez al día o en caso de un evento adverso severo de suspendió hasta la resolución.

\section{Inducción}

La etapa de pretratamiento consistió una dosis progresiva de prednisona como monoterapia durante 7 días (25 mg hasta $100 \mathrm{mg}$ ), para posteriormente continuar con una etapa de inducción a la remisión de 28 días de duración. En los días $+1,+8,+15$ se administró en conjunto a la prednisona; daunorrubicina $35 \mathrm{mg} / \mathrm{m}^{2}$ de superficie corporal y vincristina $1,2 \mathrm{mg} / \mathrm{m}^{2}$ (máximo 2 $\mathrm{mg}$ ). Al finalizar las 4 semanas de tratamiento se realizó estudio de médula ósea para evaluar la respuesta al tratamiento o la falla terapéutica (refractariedad o muerte). Se consideró leucemias refractarias a aquellas con más de $5 \%$ de blastos posterior a las primeras 4 semanas de tratamiento al igual que recaída a aquellos casos que integraron una $\mathrm{RC}$ y durante el seguimiento incrementaron el conteo de blastos por encima de $5 \%$ de blastos. 
Tabla 1. Protocolo institucional para el tratamiento de la leucemia linfoide del adulto

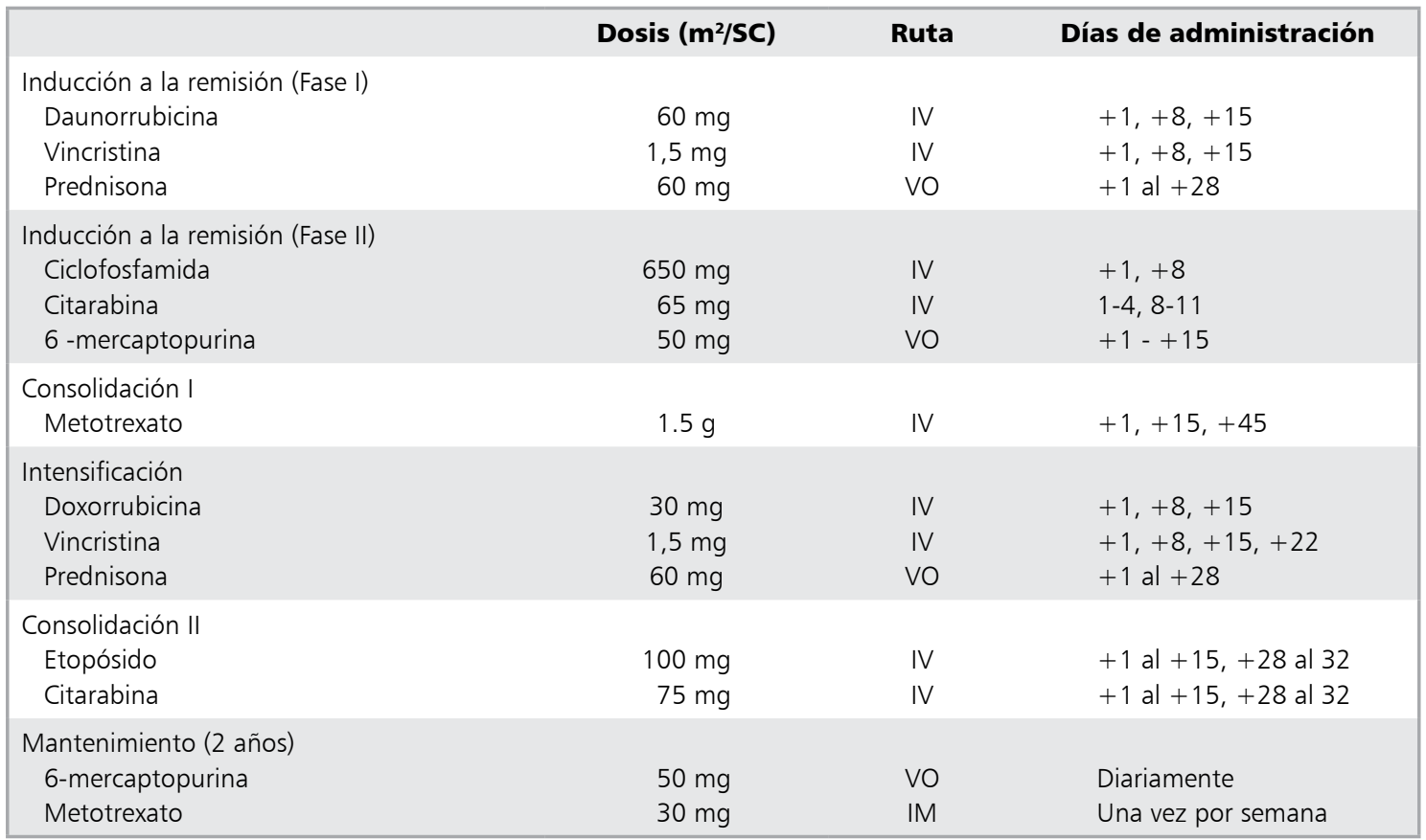

SC: Superficie corporal, IM: intramuscular, IV: intravenosa, VO: Vía oral. Al final de cada bloque de tratamiento se administra quimioterapia intratecal con metotrexato $15 \mathrm{mg}$; durante el mantenimiento la quimioterapia intratecal se administra bimensual.

\section{Consolidación y mantenimiento}

La consolidación fue realizada mediante bloques secuenciales enfocados en la protección del sistema nervioso central. En caso de contar con un donador HLA compatible se refirió al paciente al área de trasplante de progenitores hematopoyéticos. La duración del mantenimiento fue de 2 años mediante la administración de 6-mercaptopurina (50 mg por vía oral diariamente) y dosis intramuscular de metotrexato semanal ( $50 \mathrm{mg}$ ). La dosis de metformina se mantuvo a $850 \mathrm{mg}$ tres veces al día, solo ajustándola según a la tolerancia gástrica.

\section{Análisis estadístico}

Se utilizó el software estadístico SPSS versión 20.0 (IBM, Chicago ILL, USA). Para el análisis de supervivencia se utilizó el método de KaplanMeier, y la diferencia entra los diferentes grupos se realizó mediante el test de log-rank. El riesgo de las diferentes variables sobre la refractariedad al igual que la recaída se realizó mediante la estimación del riesgo relativo $(\mathrm{RR})$, considerándose significativo un valor de $\mathrm{p}<0,05$. El impacto de las diferentes variables sobre el pronóstico se realizó mediante el análisis de regresión de Cox.

\section{Consideraciones éticas}

Todos los pacientes contaron con consentimiento informado de la institución previo al inicio del estudio. El número de registro dentro de la Institución es D1/15/103/03/57. El registro establecido en clinicaltrials.gov es NCT03118128.

\section{Resultados}

Se estudiaron un total de 123 pacientes con el diagnóstico de LLA de novo atendidos en el Departamento de Hematología del Hospital General de México, en la Ciudad de México. El diagnóstico se confirmó mediante citometría de flujo, excluyendo aquellos casos con estirpe $\mathrm{T}$ o la expresión de algún marcador mieloide (LLA My+). La media de edad fue de 33 años (17-79), sin mostrarse diferencias entre los dos grupos $(\mathrm{p}=0,02)$. Al estratificar en cuanto a edad, solo $39 \%(n=48)$ 
correspondió al grupo de mayores de 30 años, sin mostrarse diferencias en la distribución acorde al tipo de tratamiento. En cuanto a la cuenta de leucocitos (media de 41,2 rango: 0,2-443) 33\% $(\mathrm{n}=41)$ se consideró como de riesgo alto debido a una cuenta mayor de $30 \times 10^{3} / \mathrm{mcl}$. A pesar de no existir una diferencia significativa en cuanto a la cuenta de leucocitos entre los grupos (36,3 vs $46,1 \times 10^{3} / \mathrm{mcl}$ ), el brazo de metformina contó con un mayor número de pacientes que iniciaron su tratamiento con conteos superiores a $30 \times 10^{3} / \mathrm{mcl}$ [25 (41,1\%) vs $16(25,8 \%)]$. Tampoco se registraron diferencias entre el valor de hemoglobina, al igual que en el conteo de plaquetas. Al combinar las variables de riesgo, $61,8 \%$ de los pacientes fueron considerados como riesgo elevado de recaída $(n=76)$. De estos, la mayoría se encontraron el brazo con metformina $[41(67,2 \%)$ vs $35(46,1)]$. Todos los pacientes estudiados contaron con feno- tipo B y sin infiltración a sistema nervioso central al diagnóstico. Se excluyeron también a pacientes que pudieran contar con situaciones de riesgo para desarrollar complicaciones metabólicas, como síndrome de lisis tumoral, fracaso renal agudo o falla hepática aguda, Tabla 2.

\section{Resultados del tratamiento}

Los 123 pacientes iniciaron inducción a la remisión, 61 recibieron en conjunto con su esquema de quimioterapia con metformina ( $850 \mathrm{mg} \mathrm{PO}$ cada $8 \mathrm{~h}$ ), la evaluación de la respuesta se realizó en el día +28 de tratamiento considerándose RC si contaba con menos de $5 \%$ de blastos en médula ósea y recuperación de los valores de plaquetas y hemoglobina. En caso de contar solo con la ausencia de blastos, pero sin recuperación de los valores de neutrófilos y plaquetas, se consideró como una respuesta parcial (RP). Posterior a las 4 semanas de

Tabla 2. Parámetros clínicos de los pacientes con LLA tratados con y sin metformina

\begin{tabular}{|c|c|c|c|c|}
\hline & $\begin{array}{c}\text { Total } \\
n=123\end{array}$ & $\begin{array}{c}\text { Sin metformina } \\
n=62\end{array}$ & $\begin{array}{l}\text { Con metformina } \\
\qquad n=61\end{array}$ & $\mathbf{p}$ \\
\hline \multicolumn{5}{|l|}{ Género (\%) } \\
\hline Masculino (\%) & $71(57,7)$ & $37(52,1)$ & $34(47,9)$ & \\
\hline Femenino (\%) & $52(42,3)$ & $25(48,1)$ & $27(51,9)$ & \\
\hline Edad (años) & $33(17-79)$ & $34(17-68)$ & $33(17-79)$ & 0,702 \\
\hline Edad <30 años (\%) & $75(61)$ & $37(59,7)$ & $38(62,3)$ & \\
\hline Edad >30 años (\%) & $48(39)$ & $25(40,3)$ & $23(37,7)$ & \\
\hline Cuenta de leucocitos $\left(\times 10^{3} / \mathrm{mcl}\right)$ & $41,2(0,2-443)$ & $36,3(0,2-443)$ & $46,1(1-300)$ & 0,440 \\
\hline$<30 \times 10^{3} / \mathrm{mcl}$ & $82(66,7)$ & $46(74,2)$ & $36(59)$ & \\
\hline$>30 \times 10^{3} / \mathrm{mcl}$ & $41(33,3)$ & $16(25,8)$ & $25(41)$ & \\
\hline Hemoglobina (g/dl) & $11,6(1,7-14,5)$ & $8,3(2,3-14,1)$ & $8,2(1,7-14,5)$ & 0,764 \\
\hline Plaquetas (x 103/mcl) & $40,1(2-241)$ & $35,6(3-195)$ & $44,6(2-241)$ & 0,263 \\
\hline \multicolumn{5}{|l|}{ Tipo de riesgo } \\
\hline Riesgo alto & $76(61,8)$ & $35(46,1)$ & $41(67,2)$ & \\
\hline Riesgo habitual & $47(38,2)$ & $27(43,5)$ & $20(32,8)$ & \\
\hline \multicolumn{5}{|l|}{ Respuesta al tratamiento } \\
\hline Remisión completa (\%) & $89(72,4)$ & $41(66,1)$ & $48(78,7)$ & \\
\hline Muerte en inducción (\%) & $13(10,6)$ & $07(11,3)$ & $06(9,8)$ & \\
\hline Refractariedad (\%) & $21(17,0)$ & $14(22,6)$ & $07(11,5)$ & \\
\hline \multicolumn{5}{|l|}{ Respuesta general en inducción } \\
\hline Respuesta & $86(71,7)$ & $40(65,6)$ & $46(78)$ & \\
\hline Falla terapéutica a las 4 semanas & $34(28,3)$ & $21(34,4)$ & $13(22)$ & \\
\hline Recaídas & $29(23,6)$ & $21(17,1)$ & $08(6,5)$ & \\
\hline Recaída temprana & $17(13,8)$ & $14(22,6)$ & $03(4,9)$ & \\
\hline Recaída tardía & $12(09,8)$ & $07(11,3)$ & $05(8,2)$ & \\
\hline Remisión completa & $94(76,4)$ & $41(66,1)$ & $53(86,9)$ & \\
\hline
\end{tabular}


tratamiento, $72,4 \%(\mathrm{n}=89)$ integraron $\mathrm{RC}, 10,6 \%$ $(\mathrm{n}=13)$ fallecieron durante la etapa de inducción y $17 \%(n=21)$ se consideraron como leucemias refractarias. El porcentaje de fallas terapéuticas a las 4 semanas fue de $28,3 \%(n=34)$, siendo mayor en el grupo de quimioterapia en comparación con el grupo al que se le adicionó MET $(34,4 \%$ vs $22,2 \%)$. La principal causa de muerte fue secundaria a procesos infecciosos durante el período de neutropenia, reportándose solo dos casos de hemorragia en sistema nervioso central. En el grupo de metformina, la mayor parte de los eventos adversos fueron grado I/II, siendo las alteraciones gastrointestinales (náusea, diarrea) las principales. En $20 \%$ de los casos se registró elevación transitoria de las enzimas hepáticas, pero no rebasando 2 veces el valor limítrofe. No se registró ningún caso de acidosis láctica asociada a metformina.

En cuanto a su perfil de seguridad, la mayor parte de los eventos adversos fueron tolerables y no obligaron a la suspensión del fármaco.

\section{Supervivencia global y recaída}

La media de seguimiento fue de 469 días (período máximo de 778 días), la supervivencia global a 700 días de seguimiento fue de $43 \%$, con una supervivencia libre de enfermedad de $47 \%$ (Figuras 1,2). Durante la inducción a la remisión, la mayoría de las muertes ocurrieron en las primeras dos semanas, pero durante el seguimiento estas fueron asociadas principalmente a las recaídas. Durante el seguimiento, la frecuencia de recaídas fue de 26,3\% ( $n=29)$, siendo en su mayoría tempranas $(\mathrm{n}=17,13,8 \%)$. En cuanto a los grupos

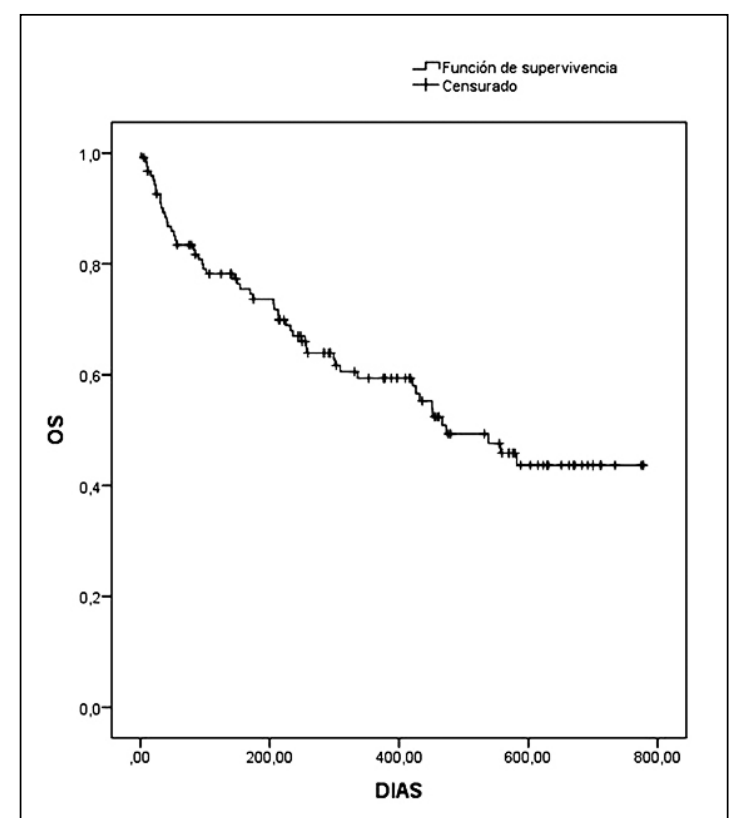

Figura 1. Supervivencia global en pacientes con LLA. La media de seguimiento fue de 469 días (rango máximo de 778 días), la supervivencia global a 70 días de seguimiento fue de $43 \%$, con una supervivencia libre de enfermedad de $47 \%$.

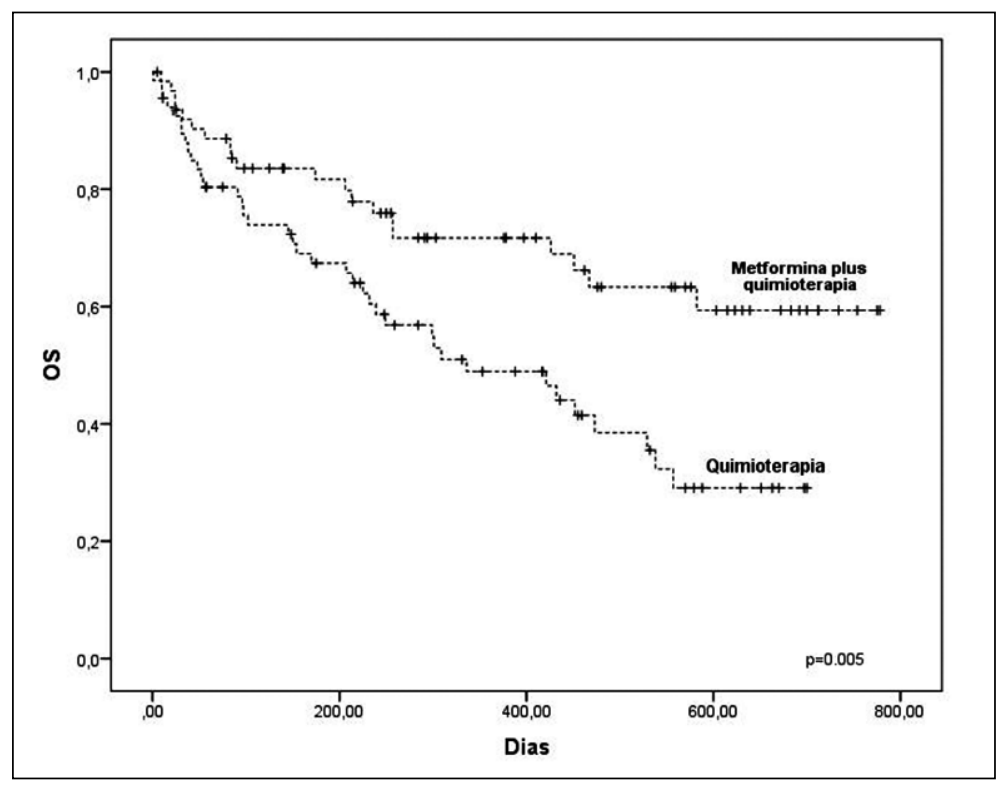

Figura 2. Supervivencia global en pacientes con LLA con y sin metformina. La media de seguimiento fue de 469 días (rango máximo de 778 días), la supervivencia global fue de $68,3 \%$ con metformina y de $43,3 \%$ sin metformina. 
Tabla 3. Estimación de riesgo de las diferentes variables de estudio

\begin{tabular}{|lcccccc|}
\hline & \multicolumn{3}{c}{ Falla terapéutica } & & \multicolumn{2}{c|}{ Recaída } \\
Variable de estudio & RR (95\%IC) & Rango & Valor $\mathbf{p}$ & RR (95\%IC) & Rango & Valor p \\
\hline Edad $>35$ años & 1,553 & $0,884-2,728$ & 0,094 & 0,822 & $0,418-1,614$ & 0,569 \\
\hline Leucocitos $>30 \times 10^{3} / \mathrm{mcl}$ & 1,090 & $0,603-1,972$ & 0,467 & 0,761 & $0,369-1,569$ & 0,303 \\
\hline Riesgo alto & 1,299 & $0,701-2,409$ & 0,263 & 0,876 & $0,460-1,667$ & 0,424 \\
\hline Género masculino & 0,831 & $0,471-1,467$ & 0,332 & 1,198 & $0,619-2,317$ & 0,374 \\
\hline Metformina & 0,640 & $0,351-1,156$ & 0,096 & 0,387 & $0,185-0,806$ & $0,006^{*}$ \\
\hline Quimioterapia sola & 1,562 & $0,864-2,824$ & 0,096 & 2,582 & $1,240-5,378$ & 0,006 \\
\hline
\end{tabular}

de tratamiento, los pacientes que se encontraban bajo tratamiento con metformina registraron una tasa menor de recaídas en comparación con el grupo que recibió solamente quimioterapia $(6,5 \%$ vs $17,1 \%, p=0,006)$. Semejante a esto, la supervivencia global también fue mayor en los pacientes que recibieron tratamiento con metformina $(\mathrm{p}=0,009)$.

\section{Factores pronósticos asociados}

Tanto la edad como la cifra de leucocitos al diagnóstico son los principales factores pronósticos clínicos. Estos al combinarse permiten identificar a los casos con un riesgo elevado de recaída. Al analizar los diferentes factores, aquellos pacientes los cuales solo recibieron quimioterapia mostraron un mayor riesgo de recaída en comparación con los que recibieron metformina.

En cuanto al tipo de tratamiento, aquellos pacientes a los que solo se les administró únicamente quimioterapia mostraron un mayor riesgo de recaída (RR: 2,582 (1,240-5,378), p = 0,006) en comparación con aquellos que recibieron en conjunto metformina (RR: $0,387(0,185-0,806)$ 95\% IC). La asociación de los diferentes factores de riesgo sobre la refractariedad o las recaídas se describe en la Tabla 3.

\section{Discusión}

El éxito del tratamiento de la leucemia linfoblástica aguda del adulto se basa tanto en la combinación de diversos fármacos conocidos como quimioterapia en combinación con otras estrategias como son los anticuerpos monoclonales (anti-CD20, anti-CD22 o CD19) o fármacos inhibidores de proteínas con actividad de tirosina-cinasa como la cinasa BCR-ABL1 (imatinib, nilotinib, dasatinib ${ }^{25,26}$. Estos biomarcadores tanto moleculares como por citometría de flujo han permitido personalizar y mejorar el rendimiento de las diferentes quimioterapias ${ }^{27,28}$.

Debido a esto y con la finalidad de reducir los sesgos, en este estudio se excluyeron aquellos pacientes que requirieran combinar la quimioterapia con cualquier otro agente o que al momento del diagnóstico ya contaran con marcadores de riesgo elevado de recaída (LLA- T, LLA My+). $\mathrm{Al}$ analizar la eficacia y seguridad sobre un grupo específico de pacientes, la adición de metformina al tratamiento se asoció con un incremento en el porcentaje de remisiones completas, así como una disminución en el porcentaje de leucemias refractarias a tratamiento. El segundo objetivo fue evaluar el efecto sobre las recaídas, semejante a lo sucedido durante la inducción a la remisión, el porcentaje de leucemias refractarias en el grupo de pacientes tratados con metformina fue menor, se asoció con una mejoría de la supervivencia siendo esta diferencia mayor al considerar el porcentaje recaídas tempranas. Estos datos sugieren que la adición de metformina puede ofrecer nuevas oportunidades para el tratamiento de las leucemias agudas en especial en aquellas consideradas como de alto riesgo de recaída ${ }^{29,30}$. La evidencia en diferentes líneas celulares ha permitido identificar los efectos de metformina sobre la proliferación tumoral o la apoptosis ${ }^{31}$. Inicialmente la mayor parte de los ensayos solo consideraba el arresto de las células en etapa $\mathrm{G} 0 / \mathrm{G} 1$, pero posteriormente se identificó que su adición bloquea importantes vías de señalización intracelulares implicadas en la supervivencia celular como lo son la vía PI3K/ 
$\mathrm{AKT} / \mathrm{m} \mathrm{TOR}^{32-34}$. Un segundo mecanismo intracelular identificado fue la activación de diversas proteínas implicadas en vías energéticas como lo son la AMPK, esta cinasa es de extrema importancia debido a que mantiene el balance energético de las células en especial baja situaciones que condicionen un daño celular ${ }^{35}$. La actividad enzimática de la AMPK depende de los diferentes requerimientos energéticos de cada célula incluyendo las células tumorales, al inducir su actividad (por ejemplo, mediante el uso de metformina) el metabolismo se transforma a un estado catabólico promoviendo finalmente la apoptosis ${ }^{36,37}$. Cada vez se conoce más acerca de las implicaciones metabólicas sobre la génesis o la proliferación tumoral e inclusive considerándose como un mecanismo independiente $^{38}$. Esto sugiere que al considerar todos los mecanismos antitumorales de metformina, la mayoría se deriva de la activación de AMPK y de ahí se deriva no solo el bloqueo energético sino de diferentes vías de señalización implicadas tanto en la proliferación como la evasión de la apoptosis ${ }^{39}$. Finalmente, la adición de metformina se asoció con mejores resultados que un régimen estándar de quimioterapia para el tratamiento de la leucemia linfoide del adulto. Debido a que en América Latina es limitado el acceso a nuevos fármacos, como la inmunoterapia dirigida, el uso de este tipo de drogas de bajo costo y accesibles podría mejorar el rendimiento de los diferentes tipos de tratamientos oncológicos.

Lo anterior debe ser estudiado en ensayos clínicos con el diseño apropiado.

En conclusión, la adición de metformina a un régimen convencional de quimioterapia podría mejorar la respuesta inicial y reducir la probabilidad de recaídas tempranas en pacientes con leucemia linfoblástica aguda.

Agradecimientos: El MD Christian Ramos Peñafiel es estudiante del "Posgrado en Ciencias Médicas y Odontológicas de la UNAM”. Este trabajo tuvo apoyo de la Dirección de Investigación Hospital General México HGM/DI/08/204/04/17, D1/15/103/03/57.

\section{Referencias}

1. Aldoss IT, Marcucci G, Pullarkat V. Treatment of Acute Lymphoblastic Leukemia in Adults: Applying Lessons
Learned in Children. Oncology (Williston Park) 2016; 30 (12): 1080-91.

2. Terwillger T, Abdul-Hay M. Acute lymphoblastic leukemia: a comprehensive review and 2017 update. Blood Cancer J 2017; 7 (6): e577.

3. Thomas X, Le Jeune C. Treating adults with acute lymphocytic leukemia: new pharmacotherapy options. Expert Opin Pharmacother 2016; 17: 2319-30.

4. Paul S, Kantarjian H, Jabbour EJ. Adult Acute Lymphoblastic Leukemia. Mayo Clin Proc 2016; 91 (11): 1645-66.

5. Kansagra A, Litzow M. Treatment of Young Adults with Acute Lymphoblastic Leukemia. Curr Hematol Malig Rep 2017; 12 (3): 187-96.

6. Kantarjian H, Stein A, Gökbuget N, Fielding AK, Schuh AC, Ribero JM, et al. Blinatumomab versus Chemotherapy for Advanced Acute Lymphoblastic Leukemia. NEJM 2017; 376 (9): 836-47.

7. Lee KJ, Chow V, Weissman A, Tulpule S, Aldoss I, Akhtari M. Clinical use of blinatumomab for B-cell acute lymphoblastic leukemia in adults. Ther Clin Risk Manag 2016; 12: 1301-10.

8. Shaughnessy AF. Monoclonal antibodies: magic bullets with a hefty price tag. BMJ 2012; 345: e8346.

9. Hladnik L, Augustin K, DeFrates S. Advancements in Therapy for Acute Lymphoblastic Leukemia: Blinatumomab. J Adv Pract Oncol 2016: 7 (1): 76-82.

10. Peng M, Darko KO, Tao T, Huang Y, Su Q, He C, et al. Combination of metformin with chemotherapeutic drugs via different molecular mechanisms. Cancer Treat Rev 2017; 54: 24-33.

11. Mukai S, Moriya S, Hiramoto M, Kazama H, Kokuba H, Che XF, et al. Macrolides sensitize EGFR-TKI-induced non-apoptotic cell death via blocking autophagy flux in pancreatic cancer cell lines. Int J Oncol 2016; 48 (1): 45-54.

12. Mei Z, Liang M, Li L, Zhang Y, Wang Q, Yang W. Effects of Statinson Cancer Mortality and Progression: A Systematic Review and Meta-analysis of 95 Cohorts including 1,111,407 Individuals. Int J Cancer 2017; 140 (5): 1068-81.

13. Fuchs C, Meyerhardt JA, Heseltine DL, Niedzwiecki D, Hollis D, Chan AT, et al. Influence of regular aspirin use on survival for patients with stage III colon cancer: Findings from Intergroup trial CALGB 89803. J Clin Oncol 2005; 23 (16 suppl): 3530.

14. Daugan M, DufaÿWojcicki A, D’Hayer B, Boudy V. Metformin: An anti-diabetic drug to fight cancer. Pharmacol Res 2016; 113 (Pt A): 675-85.

15. Leone A, Di Gennaro E, Bruzzese F, Avallone A, Budillon A. New perspective for an old antidiabetic drug: 
metformin as anticancer agent. Cancer Treat Res 2014; 159: 355-76.

16. Evans JM, Donnelly LA, Emslie-Smith AM, Alessi DR, Morris AD. Metformin and reduced Risk of cancer in diabetic patients. BMJ 2005; 330 (7530): 1304-5.

17. Libby G, Donnelly LA, Donnan PT, Alessi DR, Morris $\mathrm{AD}$, Evans JM. New users of metformin are at low risk of incident cancer: a cohort study among people with type 2 diabetes. Diabetes Care 2009; 32 (9): 1620-5. doi: $10.2337 / \mathrm{dc} 08-2175$.

18. Peng M, Darko KO, Tao T, Huang Y, Su Q, He C, et al. Combination of Metformin with chemotherapeutic drugs via different molecular mechanism. Cancer Treat Rev 2017; 54: 24-33.

19. Tan MH, Alquraini H, Mizokami-Stout K, MacEachern M. Metformin: From Research to Clinical Practice. Endocrinol Metab Clin North Am 2016; 45 (4): 819-43.

20. Dowling RJ, Niraula S, Stambolic V, Goodwin PJ. Metformin in cancer: translational challenges. J Mol Endocrinol 2012; 48 (3): R31-43.

21. Wu W, Yang JL, Wang YL, Wang H, Yao M, Wang L, et al. Reversal of multidrug resistance of hepatocellular carcinoma cells by metformin through inhibiting NF- $\kappa \mathrm{B}$ gene transcription. World J Hepatol 2016; 8 (23): 98593.

22. Abbasi MM, Valizadeh H, Hamishehkar H, Zakeri-Milani P. Inhibition of P-glycoprotein expression and function by anti-diabetic drugs gliclazide, metformin, and pioglitazone in vitro and in situ. Res Pharm Sci 2016; 11 (3): 177-86.

23. Pan J, Chen C, Jin Y, Fuentes-Mattei E, Velazquez-Tores G, Benito JM, et al. Differential impact of structurally different anti-diabetic drugs on proliferation and chemosensitivity of acute lymphoblastic leukemia cells. Cell Cycle 2012; 11 (12): 2314-26.

24. Ramos-Peñafiel CO, Martínez-Murillo C, Santoyo-Sánchez A, Jiménez-Ponce F, Rozen-Fuller E, Collazo-Jaloma J, et al. Effect of Metformin addition to an acute lymphoblastic Leukemia chemotherapy treatment. Rev Med Inst Mex Seguro Soc 2014; 52 (3): 270-5.

25. Rowe JM, Buck G, Burnett AK, Chopra R, Wiernik PH, Richards SM, et al. Induction therapy for adults with acute lymphoblastic leukemia: results of more than 1500 patients fromthe international ALL trial: MRC UKALL XII/ECOG E2993. Blood 2005; 106 (12): 3760-67.

26. Frey NV, Luger SM. How I treat adults with relapsed or refractory Philadelphia chromosome-negative acute lymphoblastic leukemia. Blood 2015; 126 (5): 589-96.

27. Thomas DA, O’Brien S, Faderl S, García-Manero G, Ferrajoli A, Wierda W, et al. Chemoimmunotherapy with a modified hyper-CVAD and rituximab regimen improves outcome in de novo Philadelphia chromosome-negative precursor B-lineage acute lymphoblastic leukemia. J Clin Oncol 2010; 28 (24): 3880-9.

28. Saini L, Brandwein J. New Treatment Strateges for Philadelphia Chromosome Positive Acute Lymphoblastic Leukemia. Curr Hematol Malig Rep 2017; 12 (2): 13642.

29. Chae YK, Arya A, Malecek MK, Shin DS, Carneiro B, Chandra S, et al. Repurposing Metformin for cancer treatment: current clinical studies. Oncotarget 2016; 7 (26): 40767-80.

30. Leone A, Di Gennaro E, Bruzzese F, Avalllone A, Budilon A. New perspective for and oldanti-diabetic drug: Metformin asanti-canceragent. Cancer Treat Res 2014; 159: 355-76.

31. Daugan M, DufaÿWojcicki A, d'Hayer B, Boudy V. Metformin: An anti-diabetic drug to fight cancer. Pharmacol Res 2016; 113: 675-85.

32. Patel S, Singh N, Kumar L. Evaluation of Effects of Metformin in Primary Ovarian Cancer. Asian Pac J Cancer Prev 2015; 16: 6973-9.

33. Cai X, Hu, Tan X, Cheng W, Wang Q, Chen X, et al. Metformin Induced AMPK Activation, G0/G1 Phase Cell Cycle Arrest and the Inhibition of Growth of Esophageal Squamous Cell Carcinomas in Vitro and In Vivo. PLoS One 2015; 10 (7): e0133349.

34. Zhang T, Guo P, Zhang Y, Xiong H, Yu X, Xu S, et al. The antidiabetic drug Metformin inhibits the proliferation of bladder cancer cells in vitro and in vivo. Int J Mol Sci 2013; 14 (12): 24603-18.

35. García D, Shaw RJ. AMPK: Mechanisms of Cellular Energy Sensing and Restoration of Metabolic Balance. Mol Cell 2017; 66 (6): 789-800.

36. Guigas B, Viollet B. Targeting AMPK: From Ancient Drugs to New Small-Molecule Activators. EXS 2016; 107: 327-50.

37. Hardie DG, Ross FA, Hawley SA. AMP-activated protein kinase: a target for drugs both ancient and modern. Chem Biol 2012; 19 (10): 1222-36.

38. HanahanD, Weinberg RA. Hallmarks of cancer, the next generation. Cell 2011; 144 (5): 646-74.

39. Choi YK, Park KG. Metabolic roles of AMPK and Metformin in cancer cells. Mol Cells 2013; 36 (4): 279-87. 